\title{
Perfil de ácidos grasos y contenido energético en músculo de juveniles de cabrilla (Paralabrax humeralis) acondicionados al cautiverio
}

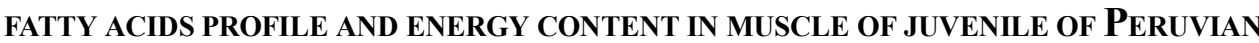 \\ ROCK SEA BASS (Paralabrax humeralis) CONDITIONED TO CAPTIVITY
}
Maryandrea Rosado-Salazar ${ }^{1.3}$, Jhon Dionicio-Acedo ${ }^{1}$, Jorge Flores-Mego ${ }^{1}$, Fabrizzio Vásquez ${ }^{2}$, Lizbeth Cosio ${ }^{1}$, Arturo Aguirre-Velarde ${ }^{1}$

\section{Resumen}

Se evaluó el perfil de ácidos grasos y contenido energético en músculo de juveniles de Paralabrax humeralis acondicionados a tres temperaturas $\left(12,17\right.$ y $\left.22^{\circ} \mathrm{C}\right)$ por un periodo de 90 días. Se utilizaron tanques de fibra de vidrio de $2 \mathrm{~m}^{3}$ conectados a sistemas de recirculación de agua con control de temperatura. Los peces recibieron dos veces al día alimento balanceado comercial (pellets) de $4 \mathrm{~mm}$ de diámetro, $42 \%$ de proteína y $12 \%$ de grasa. Cada 30 días se registró el peso y longitud de todos los peces. Además, seis peces por temperatura de aclimatación fueron sacrificados para medir las concentraciones de ácidos grasos y el contenido energético del tejido muscular. Las observaciones muestran que $P$. humeralis acepta rápidamente el alimento balanceado alcanzando una sobrevivencia del 95\% durante el periodo de evaluación. La concentración muscular de ácidos linoleico y linolénico aumentaron más del 100\% en los tres grupos de temperatura. Contrariamente, la concentración de ácidos docosahexaenoico (DHA), araquidónico (ARA), docosopentaenoico (DPA) y eicosapentaenoico (EPA) disminuyeron en 48, 45, 26 y $20 \%$, respectivamente. El contenido energético en músculo aumentó $13 \%$ durante el periodo de aclimatación. Se concluye que $P$. humeralis se adapta rápidamente a las condiciones de cautiverio; sin embargo, el alimento proporcionado debe contener mayores cantidades de ARA, EPA, DPA y DHA.

Palabras clave: perfil lipídico; aclimatación; energía; nutrición; cabrilla

\footnotetext{
${ }^{1}$ Laboratorio de Ecofisiología Acuática, Instituto del Mar del Perú (IMARPE), Callao, Perú

${ }^{2}$ Facultad de Ingeniería Económica, Estadística y Ciencias Sociales, Universidad Nacional de Ingeniería, Lima, Perú

${ }^{3}$ E-mail: mrosado@imarpe.gob.pe
}

Fuente de financiamiento: PpR Ordenamiento y Desarrollo de la Acuicultura (DGIA-IMARPE)

Recibido: 5 de julio de 2017

Aceptado para publicación: 22 de noviembre de 2017 
The fatty acids profile and energy content in muscle of juveniles of Paralabrax humeralis conditioned at three temperatures $\left(12,17\right.$ and $\left.22^{\circ} \mathrm{C}\right)$ for a period of 90 days was evaluated. For this purpose, $2 \mathrm{~m}^{3}$ fiberglass tanks connected to water recirculation systems with temperature control were used. The fishes were fed with commercial balanced feed (pellets) of $4 \mathrm{~mm}$ diameter, $42 \%$ protein and $12 \%$ fat twice a day. Every 30 days the weight and length of all fishes was recorded. In addition, six fish per acclimation temperature were sacrificed to measure fatty acids concentrations and energy content of muscle tissue. The observations showed that $P$. humeralis quickly accepts the artificial feed reaching a $95 \%$ survival during the evaluation period. The muscle concentration of linoleic and linolenic acids increased more than $100 \%$ in the three temperature groups. Contrarily, the concentration of docosahexaenoic acid (DHA), arachidonic acid (ARA), docosapentaenoic acid (DPA) and eicosapentaenoic acid (EPA) decreased by 48, 45, 26 and $20 \%$ respectively. The energy content in muscle increased $13 \%$ during the conditioning period. It is concluded that $P$. humeralis adapts quickly to the conditions of captivity; however, the feed provided must contain higher amounts of ARA, EPA, DPA and DHA.

Key words: lipid profile; acclimation; energy; nutrition; Peruvian rock sea bass

\section{INTRODUCCIÓN}

La cabrilla (Paralabrax humeralis, Valenciennes 1828) es un Serránido de importancia comercial en las costas del Perú, alcanzando desembarques de hasta $2481 \mathrm{TM}$ (Produce, 2015). Habita en áreas costeras rocoso-arenosas desde Ecuador ( $\left.3^{\circ} 00^{\prime} \mathrm{S}\right)$ hasta la región austral de Chile $\left(33^{\circ} 10^{\prime} \mathrm{S}\right)$ (Chirichigno y Vélez, 1998). Se le encuentra desde aguas someras hasta los $200 \mathrm{~m}$ de profundidad, donde la temperatura del agua va desde 13.6 hasta $17.2^{\circ} \mathrm{C}$ y con contenido de oxígeno desde 0.34 a $2.77 \mathrm{ml}^{-1} \mathrm{l}^{-1}$ (Miñano y Castillo 1971; Goicochea et al., 2014).

Esta especie llega a medir hasta 54.5 $\mathrm{cm}$ de longitud total y pesar hasta $2.5 \mathrm{~kg}$ (Medina et al., 2004; Goicochea et al., 2014). Debido a que es una especie con gran demanda en el mercado nacional, se promueve la investigación sobre su biología con el fin de generar tecnologías para su cultivo. Si bien, sobre esta especie se han desarrollado estudios sobre sus hábitos alimenticios (Miñano y Castillo, 1971; Medina et al., 2004), taxonomía (Chirichigno y Vélez, 1998, Rodrigo y Pequeño, 2001), escala de madu- rez gonadal y hermafroditismo (género Paralabrax; Oliva et al., 1992; Sadovy y Domeier, 2005; Sánchez y Gálvez, 2015), no se disponen de experiencias de aclimatación al cautiverio y estudios de requerimientos nutricionales.

Los peces obtienen los nutrientes necesarios para su desarrollo y crecimiento de sus presas en ambientes naturales; sin embargo, en cautiverio se requiere conocer los requerimientos nutricionales y energéticos para elaborar dietas adecuadas que garanticen un buen crecimiento en sistemas de cultivo. Es conocido que los peces carnívoros marinos necesitan cantidades importantes de ácidos grasos insaturados (HUFA) para su crecimiento, desarrollo y reproducción (Sargent et al., 1993; Sargent, 1995; Ghioni et al., 1999; Villarreal, 2011; Caballero et al., 2002; Bell y Sargent, 2003; Tidwell et al., 2007; López et al., 2009). Entre ellos, los de mayor importancia fisiológica son el ácido docosahexaenoico (DHA, 22:6n-3), el ácido eicosapentaenoico (EPA, 20: 5n-3) y el ácido araquidónico (ARA, 20: 4n-6) por ser componentes estructurales de los fosfolípidos de la membrana celular (Sargent et al., 1999). 
La temperatura ambiental es una de las variables de mayor influencia en los procesos metabólicos de animales poiquilotermos como los peces; afectando el conjunto del balance energético del organismo (Cho y Kaushik, 1990). En este contexto, los objetivos del presente estudio fueron describir el proceso de aclimatación de $P$. humeralis a condiciones de cautiverio y evaluar la evolución del perfil de ácidos grasos y contenido energético en músculo de juveniles de esta especie durante su aclimatación a diferentes temperaturas.

\section{Materiales y Métodos}

\section{Captura y Acondicionamiento al Cauti- verio}

Se capturaron 500 juveniles $(12-15 \mathrm{~cm}$ de longitud total) de cabrilla en la Isla San Lorenzo (12 $\left.{ }^{\circ} 6^{\prime} 44^{\prime \prime} \mathrm{S}-77^{\circ} 11^{\prime} 48^{\prime \prime} \mathrm{O}\right)$ en febrero de 2014 (verano austral), utilizando red de pesca tipo cerco, con malla de $17 \mathrm{~mm}$ manipulada bajo el agua por dos buzos. Los peces capturados fueron trasladados en tanques de 5001 con aireación constante al Laboratorio de Ecofisiología Acuática en la sede central del Instituto del Mar del Perú (IMARPE) en un tiempo estimado de dos horas. En el laboratorio se separaron los peces muertos o que presentaban lesiones, así como a los individuos de la especie Paralabrax callensis, la cual presenta similitudes morfológicas con los individuos de P. humeralis.

El periodo de acondicionamiento de los peces a condiciones de cautiverio tuvo una duración de 90 días. Este se llevó a cabo en un tanque circular de fibra de vidrio de $10 \mathrm{~m}^{3}$ conectado a un sistema de recirculación con aireación constante, temperatura a $19 \pm 1{ }^{\circ} \mathrm{C}$ y fotoperiodo 10:14 (luz: oscuridad). Se realizaron cambios del $50 \%$ de agua dos veces al día (10:00 y 17:00 horas). La alimentación fue realizada dos veces al día (09:00 y 16:00 horas) con una dieta comercial para peces (alimento peletizado) de $4 \mathrm{~mm}$ de diámetro con $42 \%$ de proteína y $12 \%$ de grasa. La temperatura $\left({ }^{\circ} \mathrm{C}\right)$ y la saturación de oxígeno disuelto (\%) fueron monitoreadas diariamente con un oxímetro HACH HQ 30d.

\section{Perfil de Ácidos Grasos y Contenido Energético}

Luego del periodo inicial de acondicionamiento al cautiverio, los peces fueron distribuidos en tanques a temperaturas de 12 , 17 y $22^{\circ} \mathrm{C}$. Cada tanque contó con 50 peces $\mathrm{y}$ dos réplicas durante un periodo experimental de 90 días. Para este fin, se utilizaron tanques circulares de $2 \mathrm{~m}^{3}$ conectados a sistemas de recirculación con control de temperatura. Las condiciones de aireación, fotoperiodo y alimentación fueron mantenidas con respecto a la aclimatación inicial.

Se realizaron muestreos al inicio y luego cada $30 \pm 1$ días, registrándose el peso húmedo (g) y la longitud total $(\mathrm{cm})$ de la totalidad de los peces en cada tratamiento de temperatura. Además, seis peces de cada réplica de temperatura fueron sacrificados para los análisis de determinación de ácidos grasos y contenido energético en músculo.

\section{Ácidos Grasos}

Los peces fueron sacrificados por exposición a sobredosis de tricaína $(0.8 \mathrm{~g}$ en 10 1 de agua). Se extrajo una porción del músculo (entre 200-250 mg) a la altura de la aleta dorsal. La muestra se guardó a $-80^{\circ} \mathrm{C}$ hasta el momento de realizar el análisis. Para la determinación de trece ácidos grasos se utilizó el método descrito por Ichihara y Fukubayashi (2010), utilizando un cromatógrafo de gases Varian CP 3800. Los resultados se expresan en miligramos de ácido graso.mg lípido ${ }^{-1}$. 


\section{Contenido Energético}

Los peces fueron colocados en la estufa para el secado a $80^{\circ} \mathrm{C}$ por 48 horas. Se tomó una porción de músculo, el cual se homogenizó para formar un pellet, con un rango de peso entre 25 y $200 \mathrm{mg}$. El pellet se colocó dentro de una bomba semi-micro calorimétrica para su combustión. La determinación se realizó por calorimetría directa, utilizando una bomba semi-micro calorimétrica Parr 6725. Cada muestra fue analizada por duplicado. Los resultados se expresan en cal.g ${ }^{-1}$ en base al peso seco.

\section{Análisis de Datos}

Los resultados de la talla, peso, ácidos grasos y contenido energético son presentados como medias de los individuos de cada tratamiento de temperatura. La prueba de Kolmogorov-Smirnov se realizó para verificar la normalidad de los datos, y en casos de no cumplir la normalidad se utilizó la prueba de Kruskal-Wallis para el análisis de los datos, considerando como factor al tiempo de muestreo. La prueba de Dunn fue utilizada para determinar posibles tendencias temporales.

Las observaciones de concentraciones de ácidos grasos a los 90 días (término del experimento) por efecto de las temperaturas $\left(12,17\right.$ y $\left.22^{\circ} \mathrm{C}\right)$ fueron evaluadas mediante un análisis de varianza, utilizando la prueba de Tukey para detectar diferencias entre tratamientos.

\section{Resultados}

\section{Acondicionamiento}

Paralabrax humeralis se adaptó a las condiciones de cautiverio, aceptando rápidamente (después de 3 días) el alimento balanceado, y el 95\% de individuos sobrevivió el periodo de acondicionamiento. Asimismo, los peces presentaron nado activo durante todo el periodo, distribuyéndose desde el fondo del tanque hasta la superficie. No se observaron comportamientos de agresividad ni canibalismo.

\section{Perfil de Ácidos Grasos en Músculo}

La concentración de ácido linolénico presentó una tendencia creciente significativa $(\mathrm{p}<0.05)$ durante el periodo de experimentación en temperaturas de 12 y $17^{\circ} \mathrm{C}$ (Figura 1a), aumentando de 3.12 y $4.35 \mathrm{mg} . \mathrm{g}^{-1}$ a 10.02 y $7.51 \mathrm{mg} . \mathrm{g}^{-1}$, lo que representa un aumento del 321 y $172 \%$, respectivamente. Sin embargo, en los individuos aclimatados a $22^{\circ} \mathrm{C}$, la concentración del ácido linolénico disminuyó de 5.55 a 4.73 mg.g-1, representando una disminución del $23 \%$.

La concentración de ácido linoleico en el músculo de los peces de los grupos de $17 \mathrm{y}$ $22{ }^{\circ} \mathrm{C}$ aumentó en ambos casos de $38.2 \mathrm{mg} \cdot \mathrm{g}^{-1}$ a 137.82 y $129 \mathrm{mg} \cdot \mathrm{g}^{-1}$, respectivamente. Este aumento fue significativamente mayor $(\mathrm{p}<0.05)$ con respecto a los individuos acondicionados a $12{ }^{\circ} \mathrm{C}\left(\right.$ de 36.71 a $\left.82.73 \mathrm{mg} \cdot \mathrm{g}^{-1}\right)$. El aumento de la concentración del ácido linoleico fue mayor al $100 \%$ en los tres tratamientos al término del estudio.

La concentración del ácido araquidónico muscular presentó una tendencia decreciente significativa ( $p<0.05$; Figura $1 \mathrm{c}$ ), donde los valores disminuyeron de 12.33, $13.63 \mathrm{y}$ $13.93 \mathrm{mg} . \mathrm{g}^{-1}$ a $7.03,7.09$ y $7.88 \mathrm{mg} . \mathrm{g}^{-1}$ para para las temperaturas 12,17 y $22^{\circ} \mathrm{C}$, respectivamente; lo cual equivale a una disminución del $45 \%$ de la concentración inicial para las tres temperaturas en estudio.

La concentración del ácido docosahexanoico (DHA) presentó una tendencia decreciente significativa $(\mathrm{p}<0.05$, Figura 1e). Las concentraciones para 12, $17 \mathrm{y}$ $22{ }^{\circ} \mathrm{C}$ disminuyeron de $141.49,149.73$ y 153.75 mg.g ${ }^{-1}$ a $72.56,77.61$ y 86.44 mg.g ${ }^{-1}$, respectivamente, representando una disminución promedio del $48 \%$. 

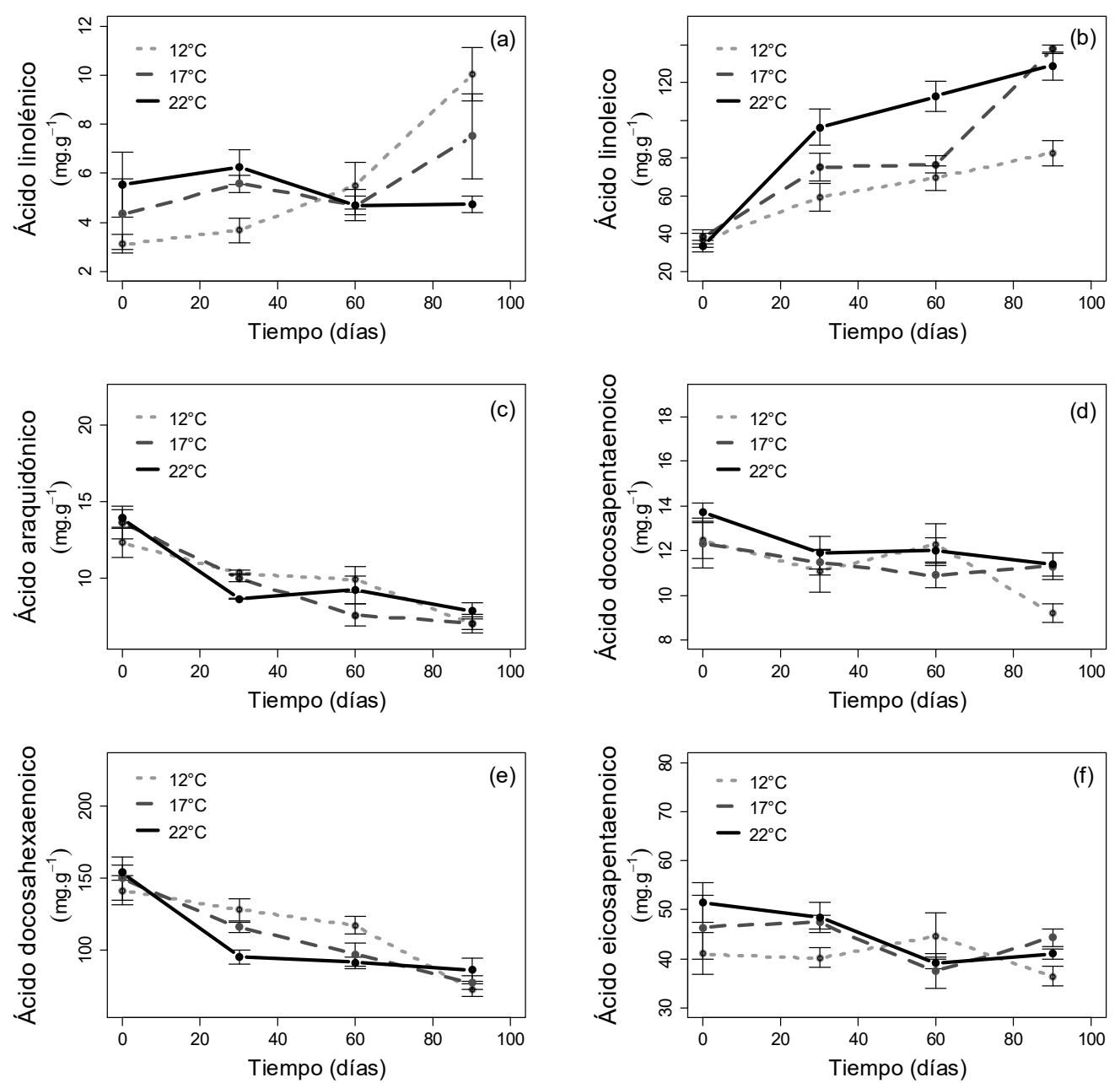

Figura 1. Evolución temporal de la concentración $\left(\mathrm{mg} \cdot \mathrm{g}^{-1}\right)$ de ácidos grasos de músculo de juveniles de cabrilla (Paralabrax humeralis) aclimatados a tres temperaturas $(12,17$ y $22{ }^{\circ} \mathrm{C}$ ): ácido linolénico (a), ácido linoleico (b), ácido araquidónico (c), ácido docosapentanoico (d), ácido docosahexaenoico (e) y ácido eicosapentaenoico (f)

Por otro lado, la concentración del ácido docosopentaenoico (DPA) no presentó cambios significativos (Figura 1d); no obstante, las concentraciones para las temperaturas de 17 y $22^{\circ} \mathrm{C}$ variaron de 12.34 y 13.7 mg. $\mathrm{g}^{-1}$ a 11.3 y $11.37 \mathrm{mg} . \mathrm{g}^{-1}$, respectivamente, siendo cambios menores que el observado a $12{ }^{\circ} \mathrm{C}$ (de 12.48 a $9.2 \mathrm{mg} . \mathrm{g}^{-1} ; \mathrm{p}<0.05$ ). Asimismo, la evolución temporal de la concentración del ácido eicosapentanoico (EPA) tampoco muestra tendencias significativas
(Figura 1f), donde las concentraciones a 12 , 17 y $22{ }^{\circ} \mathrm{C}$ disminuyeron de $41.04,46.43$ y $51.53 \mathrm{mg} . \mathrm{g}^{-1}$ a $36.46,43.33$ y $41 \mathrm{mg} . \mathrm{g}^{-1}$, respectivamente.

\section{Contenido Energético}

En el contenido energético en músculo se observó una tendencia creciente de $13 \%$ $(\mathrm{p}<0.05$; Figura 2). Los valores para $12,17 \mathrm{y}$ $22{ }^{\circ} \mathrm{C}$ aumentaron de 5205,5206 y 5257 cal.g- 


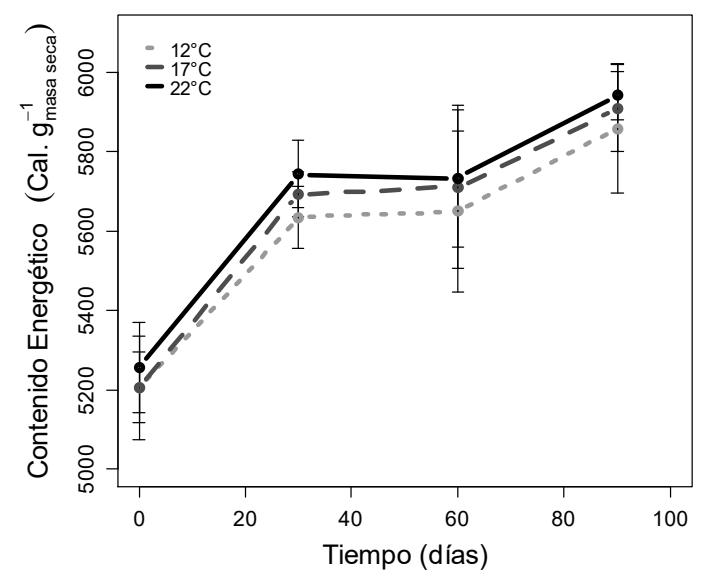

Figura 2. Evolución temporal del contenido energético en músculo de juveniles de cabrilla (Paralabrax humeralis) aclimatados a tres temperaturas

${ }^{1}$ masa seca a 5858,5909 y 5941 cal.g ${ }^{-1}$ masa seca, respectivamente, aunque sin mostrar diferencias significativas entre tratamientos.

\section{Crecimiento}

La longitud total de P. humeralis alcanzada al final del experimento fue de 21.0, 22.5 y $22.0 \mathrm{~cm}$ para las temperaturas de $12,17 \mathrm{y}$ $22{ }^{\circ} \mathrm{C}$, respectivamente. Asimismo, la ganancia de peso fue del 15,17 y $45 \%$ para los individuos acondicionados a las temperaturas 12,17 y $22{ }^{\circ} \mathrm{C}$, respectivamente. No se encontraron individuos maduros sexualmente ni se registraron desoves durante el experimento.

\section{Discusión}

El acondicionamiento relativamente rápido de la cabrilla a las condiciones de cautiverio fue evidenciado por la aceptación del alimento peletizado y el alto porcentaje de sobrevivencia. Esto concuerda con lo descrito por Mulochau y Durville (2005), quienes aclimataron individuos de la familia Serranidae en acuarios con tasas de sobrevivencia de $84.5 \%$.
Las concentraciones de los ácidos linoleico y linolénico contenidas en el músculo de los juveniles de cabrilla incrementaron, mientras que las concentraciones de DHA, EPA y ARA se redujeron durante el periodo de acondicionamiento. Es probable que los perfiles de estos ácidos grasos sean un reflejo de la composición de la dieta proporcionada (López et al., 2009). En peces marinos, los ácidos linoleico y linolénico no son precursores de ácidos grasos de cadenas largas (C20) y, por lo tanto, no son transformados en DHA, EPA o ARA (Tidwell et al., 2007). Esto debido a la ausencia de las enzimas elongasa y desaturasa, las cuales se encargan de añadir dos o cuatro átomos de carbono a los ácidos grasos esenciales e incorporar los dobles enlaces en la cadena de carbono, respectivamente (Villarreal, 2011). Por este motivo, estos ácidos grasos son esenciales en la dieta, pues no pueden ser biosintetizados. Un déficit de los ácidos grasos esenciales en la dieta podría explicar la disminución de estos en el tejido muscular a lo largo del tiempo de evaluación.

La permeabilidad de la membrana celular depende de los fosfolípidos, el colesterol y la temperatura (Hill et al., 2006), por lo cual, las variaciones de temperatura podrían generar modificaciones estructurales de los lípidos provocando una reorganización de la composición molecular y, por lo tanto, de la estructura de los fosfolípidos (Farkas et al., 2001).

Estas propiedades son aprovechadas por peces de aguas frías que requieren ácidos grasos poliinsaturados del tipo n-3, mientras que los de agua cálida requieren del tipo n-6 (Tidwell et al., 2007). Esto puede sustentarse debido a que lo ácidos grasos del tipo n6 tienen un mayor punto de fusión respecto a los del tipo n-3. En este sentido, la mayor concentración de ácido linoleico (n-6) en el músculo de los peces del estudio cultivados a 17 y $22{ }^{\circ} \mathrm{C}$ respecto de $12{ }^{\circ} \mathrm{C}$ podría estar relacionada a una reestructuración de la bicapa lipídica en función de la temperatura. 
En tal sentido, el ácido linolénico que presenta un enlace doble más, respecto al ácido linoleico, podría explicar la mayor concentración del primero en los peces cultivados a $12{ }^{\circ} \mathrm{C}$.

El aumento de la energía almacenada en tejidos como el músculo puede explicarse a través de la asimilación de alimento, cuando esta es mayor al gasto energético del organismo (Eliassen y Vahl, 1982). Hendry et al. (2000) mencionan que los peces almacenan parte de la energía ingerida como proteína muscular para ser utilizada en funciones metabólicas (mantenimiento, crecimiento, reproducción). Los peces al estar confinados a tanques de cultivo no demandaron un gasto importante de energía en búsqueda de alimento, huir de predadores, aclimatación a condiciones ambientales variables, etc. Por otro lado, las temperaturas de aclimatación a las cuales fueron expuestos los individuos de $P$. humeralis estarían dentro de su rango de tolerancia; no afectando la capacidad de esta especie para regular su balance energético (Cho y Kaushik, 1990).

\section{Conclusiones}

- Paralabrax humeralis es una especie marina que se aclimata fácilmente a las condiciones de cautiverio, aceptando el alimento y obteniendo una alta tasa de sobrevivencia.

- La alimentación proporcionada durante el periodo de experimentación evidencia claros signos de desbalance en perfil de ácidos grasos que se reflejan en la modificación de los perfiles en el músculo de $P$. humeralis.

- Se requiere realizar estudios sobre la elaboración de dietas específicas que cubran los requerimientos necesarios de los ácidos araquidónico, eicosapentaenoico, docosopentaenoico y docosahexaenoico que permitan garantizar el crecimiento y desarrollo óptimo de $P$. humeralis en sistemas de cultivo.

\section{Agradecimiento}

Este trabajo fue realizado con financiamiento del PpR Ordenamiento y Desarrollo de la Acuicultura.

\section{Literatura Citada}

1. Bell JG, Sargent JR. 2003. Arachidonic acid in aquaculture feeds: current status and future opportunities. Aquaculture 218: 491-499. doi: 10.1016/S00448486(02)00370-8

2. Caballero M, Obach A, Rosenlund G, Montero D, Gisvolt M, Izquierdo M. 2002. Impact of different dietary lipid sources on growth, lipid digestibility, tissue fatty acid composition and histology of rainbow trout, Oncorhynchus mykiss. Aquaculture 214: 253-271. doi: 10.1016/S00448486(01)00852-3

3. Chirichigno N, Vélez, J. 1998. Clave para identificar los peces marinos del Perú. 2a ed. Perú: Instituto del Mar del Perú. 496 p.

4. Cho C Y, Kaushik SJ. 1990. Nutrition energetics in fish: energy and protein utilization in rainbow trout (Salmo gairdneri). World Rev Nutr Diet 61: 132-172. doi: 10.1159/000417529

5. Eliassen J, Vahl O. 1982. Seasonal variations in biochemical composition and energy content of liver, gonad and muscle of mature and immature cod, Gadus morhua (L.) from Balsfjorden, northern Norway. J Fish Biol 20: 707-716. doi: 10.1111/j.1095-8649.1982.tb03981.x

6. Farkas T, Fodor E, Kitajka K, Halver JE. 2001. Response of fish membranes to environmental temperature. Aquaculture Res 32: 645-656. doi: 10.1046/j.1365-2109.2001.00600.x

7. Ghioni C, Tocher DR, Bell MV, Dick JR, Jargent JR. 1999. Low C18 to C20 fatty acid elongase activity and limited conversion of stearidonic acid , 18:4 (n3), to eicosapentaenoic acid , 20:5 (n-3), 
in a cell line from the turbot, Scophthalmus maximus. Biochimt Biophys Acta 1437: 170-181.

8. Goicochea C, Moquillaza $P$, Mostacero J. 2014. Edad y crecimiento de Paralabrax humeralis (Valenciennes) en el Mar del Callao, 1996. Instituto del Mar del Perú 39(1-2): 23-25.

9. Hendry A, Dittman A, Hardy R. 2000. Proximate composition, reproductive development, and a test for trade-offs in captive Sockeye salmon. T Am Fish Soc 129: 1082-1095. doi: 10.1577/15488659(2000)129<1082:PCRDAA >2.0.CO;2

10. Hill RW, Wyse GA, Anderson M. 2006. Fisiología animal. Madrid: Ed Meìdica Panamericana. $1038 \mathrm{p}$.

11. Ichihara K, Fukubayashi Y. 2010. Preparation of fatty acid methyl esters for gas-liquid chromatography. J Lipid Res 51: 635-640. doi: 10.1194/ jlr.D001065

12. López LM, Durazo E, Viana MT, Drawbridge M, Bureau DP. 2009. Effect of dietary lipid levels on performance, body composition and fatty acid profile of juvenile white seabass, Atractoscion nobilis. Aquaculture 289: 101-105. doi: 10.1016/j.aquaculture.2009.01.003

13. Medina M, Araya M, Vega C. 2004. Alimentación y relaciones tróficas de peces costeros de la zona norte de Chile. Invest Mar 32(1): 33-47. doi: 10.4067/ S0717-71782004000100004

14. Miñano J, Castillo J. 1971. Investigación biológica preliminar de la cabrilla Paralabrax humeralis (Valenciennes) en Chimbote. Lima: Informe Especial IMARPE 83. 15 p.

15. Mulochau T, Durville P. 2005. A review of the movements of fish held in captivity in the Reunion Island Aquarium over a five-year period. SPC Live Reef Fish Information Bulletin 15: 13-18.

16. Oliva ME, Bórquez AS, Olivares AN. 1992. Sexual status of Paralabrax humeralis (Serranidae) and infection by Philometra sp. (Nematoda: Dracunculidea). J Fish Biol 40: 979-980.
17. [PRODUCE] Ministerio de la Producción. 2015. Anuario estadístico pesquero y acuícola 2013. Perú: PRODUCE. $113 \mathrm{p}$.

18. Rodrigo J, Pequeño G 2001. Revisión taxonómica de especies de las subfamilies Epinephelinae y Serraninae (Pisces:Serranidae) de Chile. Rev Biol Trop 49: 157-171.

19. Sadovy Y, Domeier M. 2005. Perplexing problems of sexual patterns in the fish genus Paralabrax (Serranidae, Serraninae). J Zool 267: 121-133. doi: 10.1017/S0952836905007466

20. Sánchez J, Gálvez M. 2015. Escala de madurez gonadal de cabrilla Paralabrax humeralis (Valenciennes, 1828). Bol IMARPE 30(1-2): 3-9.

21. Sargent JR, Bell JG, Bell MV, Henderson RJ, Tocher DR. 1993. The metabolism of phospholipids and polyunsaturated fatty acids in fish. In: Lahlou B, Vitiello P (eds). Aquaculture: fundamental and applied research. Washington DC: Coastal and Estuarine Studies 43. p 103-124.

22. Sargent JR. 1995. Origins and functions of lipids in fish eggs: nutritional implications. In: Bromage NR, Roberts RJ (eds). Broodstock management and egg and larval quality. Oxford: Blackwell Science. p 353-372.

23. Sargent J, Bell G, McEvoy L, Tocher D, Estevez A. 1999. Recent developments in the essential fatty acid nutrition of fish. Aquaculture 177: 191-199. doi: 10.1016/S0044-8486(99)00083-6

24 . Tidwell JH, Coyle S, Bright LA. 2007. Effects of different types of dietary lipids on growth and fatty acid composition of largemouth bass. NA J Aquaculture 69: 257-264. doi: 10.1577/A06-040.1

25. Villarreal GE. 2011. Efecto de la concentración de HUFAs n-3 en la dieta sobre el crecimiento, supervivencia, eficiencia alimenticia e índice de condición en juveniles de Totoaba macdonaldi. Tesis de Magíster. Baja California, México: Centro de Investigación Científica y de Educación Superior de Ensenada. 62 p. 
M. Rosado et al. 